\title{
A Green function approach for the investigation of the incompressible flow past an oscillatory thin hydrofoil including floor effects
}

\author{
Eleonora Răpeanu ${ }^{1}$ and Adrian Carabineanu ${ }^{2,3 *}$
}

\author{
"Correspondence: \\ acara@fmi.unibuc.ro \\ ${ }^{2}$ Department of Mathematics, \\ University of Bucharest, Str. \\ Academiei 14, Bucharest, Romania \\ ${ }^{3}$ Institute of Mathematical Statistics \\ and Applied Mathematics of \\ Romanian Academy, Calea 13 \\ Septembrie 13, Bucharest, Romania \\ Full list of author information is \\ available at the end of the article
}

\begin{abstract}
In the framework of the small perturbations theory, we study the incompressible inviscid flow of a uniform stream past an oscillatory/undulatory thin hydrofoil including floor effects. A Green function is used to deduce the integral equation for the jump of the pressure past the foil. The integral equation is numerically solved and the average drag coefficient is calculated. For some wings there appears a propulsive force and this force increases when the hydrofoil is close to the floor.
\end{abstract}

MSC: Primary 76B10; secondary 65R20; 45H99; 31A10

Keywords: flexible hydrofoil; hypersingular integral equation; floor effect; thrust

\section{Introduction}

In the present paper we study the small-amplitude oscillatory/undulatory motion of an incompressible fluid past a thin flexible plate which performs prescribed oscillations in the presence of a wall (floor) with which it is parallel in its undisturbed state, and relative to which it is moving with constant speed. We shall limit the analysis to bodies large enough so that the Reynolds number is large. As is stated by Eloy et al. in [1], when the flexible surface has a typical speed of several body lengths per second, the flow can be considered irrotational, meaning that the flow vorticity is concentrated in thin boundary layers adjacent to the body surface and in a thin wake (vortex sheet) behind the body. Since the effects of viscosity manifest inside the thin boundary layers, we may treat the fluid as inviscid in the rest of the flow domain. Recalling Lagrange-Cauchy's theorem which states that if the flow is potential in a certain configuration, it remains potential in every configuration arising from the initial one, we deduce that the theory of the unsteady motion of lifting wings as well as the theory of potential flow can be successfully utilized (see the papers of Carabineanu [2-6], Dowell and Hall [7], Dragoș [8], Homentcovschi [9, 10], Lighthill [11], Street [12], Taylor [13], Wu [14, 15], Watkins et al. [16]).

The periodic motion of a flexible foil is oscillatory if the foil or parts of it remain rigid during the motion. The undulatory motion involves a traveling wave down the foil (Street [12]). As we know from aerodynamics and hydrodynamics studies (Dragoş and Carabineanu [17, 18], Dragoș et al. [19]), the hydrodynamic coefficients of a hydrofoil are influenced by the presence of the floor. The aim of the paper is to predict the drag or the 


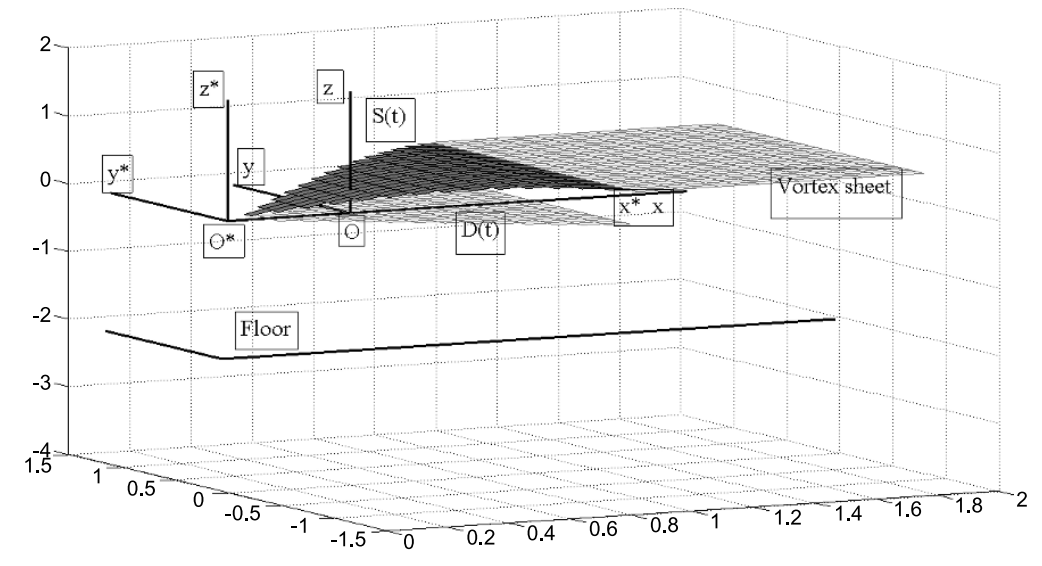

Figure 1 The flexible hydrofoil and the vortex sheet.

thrust enhancement generated by the presence of the floor. We employ like in $[2-6,8$, 9] the linearized Euler equations for the incompressible flow. For taking into account the floor effect we use the Green function of the Laplacean for the Neumann problem in the half-space. We use the integral representation for the harmonic functions and the slipping condition to obtain an integral equation for the jump of the pressure over the hydrofoil. In order to discretize the integral equation, we split the kernel of the equation into several kernels for which we provide appropriate approximation formulas depending on the type of singularity of the kernel. Assuming that the hydrofoil is subjected to harmonic oscillations, we simplify the integral equation making it independent of time. By solving the discretized integral equation we calculate the jump of the pressure over the wing.

After obtaining the pressure field, the average drag is calculate by performing a numerical integration. We study an example of undulatory motion of the flexible thin delta wing. When the frequency surpasses a critical value, the drag becomes negative i.e. it appears a propulsive force. We notice that the distance between the wing and the floor influences the drag and the thrust.

\section{The statement of the problem}

We consider the continuity and Euler equations for incompressible flow in a fixed Cartesian frame of reference $O x y z$ having the versors $\mathbf{i}, \mathbf{j}, \mathbf{k}$. At the moment $t$, the hydrofoil $S(t)$ (see Figure 1) has the equation:

$$
z-h\left(x+V_{0} t, y, t\right)=0, \quad(x, y, 0) \in D(t) .
$$

$D(t)$ is the projection of the hydrofoil onto the $O x y$ plane. We assume that

$$
\left|\frac{\partial h}{\partial x}\right| \ll 1, \quad\left|\frac{\partial h}{\partial y}\right| \ll 1, \quad\left|\frac{\partial h}{\partial t}\right| \ll 1
$$

and that the hydrofoil moves into the $O x$ direction, producing small perturbations $\mathbf{v}=$ $u \mathbf{i}+v \mathbf{j}+w \mathbf{k}$ of the vanishing velocity of the surrounding fluid. 
Let $-V_{0} \mathbf{i}$ be the average velocity of the hydrofoil $S(t)$ i.e. the velocity of $D(t)$. In a point of $D(t)$ we have

$$
x(t)=x^{*}-V_{0} t, y(t)=y^{*}, \quad\left(x^{*}, y^{*}\right) \in D^{*}=D(0) .
$$

The velocity of an arbitrary point of $(x, y, z) \in S(t)$ is

$$
\mathbf{V}=-V_{0} \mathbf{i}+\frac{d z}{d t} \mathbf{k}=-V_{0} \mathbf{i}+\frac{d h(x(t), y, t)}{d t} \mathbf{k}
$$

and the normal vector on $S(t)$ is

$$
\mathbf{n}=-\frac{\partial h}{\partial x} \mathbf{i}-\frac{\partial h}{\partial y} \mathbf{j}+\mathbf{k}
$$

We linearize the slipping condition on the two sides of $S(t)$

$$
(\mathbf{v}-\mathbf{V}) \cdot \mathbf{n}=0
$$

and we obtain

$$
w(x(t), y, \pm 0, t)=V_{0} \frac{\partial h}{\partial x}(x(t), y, t)+\frac{d h}{d t}(x(t), y, t), \quad(x(t), y, 0) \in D(t) .
$$

We consider the floor

$$
F=\left\{(x, y,-\chi) ;(x, y) \in \mathbf{R}^{2}, \chi>0\right\},
$$

where the slipping condition is also imposed:

$$
w(x, y,-\chi, t)=0 .
$$

Let $\Sigma(t)$ represent the thin vortex wake behind the hydrofoil $S(t)$. In the small-amplitude approximation theory the wake remains planar (see the demonstration of Homentcovschi in [10]). We linearize the equations of motion around the rest state (neglecting the products of the perturbation quantities) in the domain $\Xi=\{(x, y, z, t) ; t \in \mathbf{R},(x, y, z) \in \Omega(t)\}$ where $\Omega(t)=\left\{(x, y, z) \in \mathbf{R}^{3} ; z \geq-\chi\right\}-(S(t) \cup \Sigma(t))$ and we have

$$
\begin{aligned}
& \frac{\partial u}{\partial x}+\frac{\partial v}{\partial y}+\frac{\partial w}{\partial z}=0 \\
& \frac{\partial u}{\partial t}+\frac{1}{\rho_{0}} \frac{\partial p}{\partial x}=0 \\
& \frac{\partial v}{\partial t}+\frac{1}{\rho_{0}} \frac{\partial p}{\partial y}=0 \\
& \frac{\partial w}{\partial t}+\frac{1}{\rho_{0}} \frac{\partial p}{\partial z}=0
\end{aligned}
$$

where $p$ is the pressure and $\rho_{0}$ is the constant density of the fluid. The aim of the present paper is to use the boundary conditions (7), (9), and the partial differential equations (10) 
for obtaining an integral equation for the jump of the pressure across $S(t)$. In order to ensure the uniqueness of the solution we shall impose a certain periodic in time behavior to the unknowns.

\section{The Green function. The integral equation of the problem}

Eliminating $u, v, w$, from (10) we get the Laplace equation

$$
\Delta p=0 .
$$

Let us consider the Green function of the Laplacean for the Neumann problem in the half-space $z>-\chi$ :

$$
\begin{aligned}
G\left(x, y, z ; x^{\prime}, y^{\prime}, z^{\prime}\right)= & -\frac{1}{4 \pi\left|\left(x-x^{\prime}\right) \mathbf{i}+\left(y-y^{\prime}\right) \mathbf{j}+\left(z-z^{\prime}\right) \mathbf{k}\right|} \\
& -\frac{1}{4 \pi\left|\left(x-x^{\prime}\right) \mathbf{i}+\left(y-y^{\prime}\right) \mathbf{j}+\left(z+z^{\prime}+2 \chi\right) \mathbf{k}\right|} .
\end{aligned}
$$

Obviously on the floor $F$

$$
\frac{\partial G\left(x, y,-\chi ; x^{\prime}, y^{\prime}, z^{\prime}\right)}{\partial z^{\prime}}=0 .
$$

We assume that the harmonic function $p$ vanishes at infinity and we have the integral representation formula

$$
\begin{aligned}
p(x, y, z, t)= & \iint_{\partial \Omega(t)}\left[p\left(x^{\prime}, y^{\prime}, z^{\prime}, t\right) \frac{\partial G\left(x, y, z ; x^{\prime}, y^{\prime}, z^{\prime}\right)}{\partial n}-\cdots\right. \\
& \left.-\frac{\partial p\left(x^{\prime}, y^{\prime}, z^{\prime}, t\right)}{\partial n} G\left(x, y, z ; x^{\prime}, y^{\prime}, z^{\prime}\right)\right] d \sigma
\end{aligned}
$$

where $\frac{\partial}{\partial n}$ stands for the outward normal derivative and $\partial \Omega(t)=F \cup S^{+}(t) \cup S^{-}(t) \cup \Sigma^{+}(t) \cup$ $\Sigma^{-}(t)$. Here $S^{+}(t)$ and $S^{-}(t)$ are, respectively, the upper face and the lower face of $S(t)$ and similarly for $\Sigma(t)$. As usual in the small perturbation theory, we replace $S(t)$ with $D(t)$ and we assume that $\Sigma(t)$ is planar (as Homentcovschi stated in [10]). Hence

$$
\left.\frac{\partial}{\partial n}\right|_{D^{+}(t) \cup \Sigma^{+}(t)}=-\frac{\partial}{\partial z^{\prime}},\left.\quad \frac{\partial}{\partial n}\right|_{D^{-}(t) \cup \Sigma^{-}(t)}=\frac{\partial}{\partial z^{\prime}},\left.\quad \frac{\partial}{\partial n}\right|_{F}=-\frac{\partial}{\partial z^{\prime}} .
$$

From the boundary condition (7) and from the fourth equation (10) we deduce that

$$
\left.\frac{\partial p}{\partial n}\right|_{D^{+}(t) \cup \Sigma^{+}(t) \cup D^{-}(t) \cup \Sigma^{-}(t)}=0 .
$$

From (9) it follows that

$$
\left.\frac{\partial p}{\partial n}\right|_{F}=0
$$

Since the pressure is continuous over $\Sigma(t)$, from (9)-(14) we obtain

$$
p(x, y, z, t)=-\iint_{D(t)}\left[p\left(x^{\prime}, y^{\prime},+0, t\right)-p\left(x^{\prime}, y^{\prime},-0, t\right)\right] \frac{\partial G\left(x, y, z ; x^{\prime}, y^{\prime}, z^{\prime}\right)}{\partial z^{\prime}} d x^{\prime} d y^{\prime} .
$$


Taking into account that

$$
\begin{aligned}
& \frac{\partial}{\partial z} \frac{1}{\left|\left(x-x^{\prime}\right) \mathbf{i}+\left(y-y^{\prime}\right) \mathbf{j}+\left(z-z^{\prime}\right) \mathbf{k}\right|} \\
& \quad=-\frac{\partial}{\partial z^{\prime}} \frac{1}{\left|\left(x-x^{\prime}\right) \mathbf{i}+\left(y-y^{\prime}\right) \mathbf{j}+\left(z-z^{\prime}\right) \mathbf{k}\right|}, \\
& \frac{\partial}{\partial z} \frac{1}{\left|\left(x-x^{\prime}\right) \mathbf{i}+\left(y-y^{\prime}\right) \mathbf{j}+\left(z+z^{\prime}+2 \chi\right) \mathbf{k}\right|} \\
& \quad=\frac{\partial}{\partial z^{\prime}} \frac{1}{\left|\left(x-x^{\prime}\right) \mathbf{i}+\left(y-y^{\prime}\right) \mathbf{j}+\left(z+z^{\prime}+2 \chi\right) \mathbf{k}\right|},
\end{aligned}
$$

we get from the fourth equation of (10) and from (18)

$$
\begin{aligned}
& w(x, y, z, t) \\
& =\frac{1}{4 \pi} \int_{-\infty}^{t} \iint_{D\left(t^{\prime}\right)} f\left(x^{\prime}, y^{\prime}, t\right) \frac{\partial^{2}}{\partial z^{2}}\left[\frac{1}{\left|\left(x-x^{\prime}\right) \mathbf{i}+\left(y-y^{\prime}\right) \mathbf{j}+\left(z-z^{\prime}\right) \mathbf{k}\right|}-\cdots\right. \\
& \left.\quad-\frac{1}{\left|\left(x-x^{\prime}\right) \mathbf{i}+\left(y-y^{\prime}\right) \mathbf{j}+\left(z+z^{\prime}+2 \chi\right) \mathbf{k}\right|}\right] d x^{\prime} d y^{\prime} d t^{\prime},
\end{aligned}
$$

where $f(x, y, t)=\frac{p(x, y,+0, t)-p(x, y,-0, t)}{\rho_{0}}$.

In order to obtain a domain of integration that does not vary in time, we shall introduce (like in [5] and [6]) and a new system of coordinates $O^{*} x^{*} y^{*} z^{*}$, related to the lifting wing. We consider the Galilean transformations

$$
x^{*}=x+V_{0} t, \quad y^{*}=y, \quad z^{*}=z, \quad t^{*}=t .
$$

We also denote

$$
\chi^{*}=\chi, \quad x^{\prime *}=x^{\prime}+V_{0} t^{\prime}, \quad y^{*}=y^{\prime}, \quad \lambda^{*}=x^{*}-x^{\prime *}-V_{0}\left(t-t^{\prime}\right) .
$$

In the new system of coordinates, the equation of the flexible hydrofoil is

$$
z^{*}=h^{*}\left(x^{*}, y^{*}, t^{*}\right)=h\left(x+V_{0} t, y, t\right)
$$

and the perturbation velocity is

$$
\mathbf{v}^{*}\left(x^{*}, y^{*}, t^{*}\right)=\mathbf{v}\left(x+V_{0} t, y, t\right), \quad \mathbf{v}^{*}=u^{*} \mathbf{i}+v^{*} \mathbf{j}+w^{*} \mathbf{k} .
$$

Obviously,

$$
\frac{\partial h^{*}}{\partial t^{*}}=\frac{d h}{d t} .
$$

Let $D^{*}$ be the projection of the lifting surface onto the $O^{*} x^{*} y^{*}$ plane. Considering the lifting surface subject to harmonic oscillations, we impose

$$
h^{*}\left(x^{*}, y^{*}, t^{*}\right)=h^{*}\left(x^{*}, y^{*}\right) \exp \left(i \omega t^{*}\right) .
$$


In (27) $h^{*}\left(x^{*}, y^{*}\right)$ may have complex values. By convention $h^{*}\left(x^{*}, y^{*}\right) \exp \left(i \omega t^{*}\right)$ means the real part of $h^{*}\left(x^{*}, y^{*}\right) \exp \left(i \omega t^{*}\right)$. For the sake of simplicity, we shall calculate (as is usual in the oscillatory hydrofoil theory) complex values for the jump of pressure and then we shall consider the real part.

We also have

$$
f\left(x^{*}, y^{*}, t^{*}\right)=f\left(x^{*}, y^{*}\right) \exp \left(i \omega t^{*}\right) \text {. }
$$

From (7), (26), and (27) we get

$$
w^{*}\left(x^{*}, y^{*}, \pm 0, t^{*}\right)=V_{0} \frac{\partial h^{*}}{\partial x^{*}} \exp \left(i \omega t^{*}\right)+i \omega h^{*}\left(x^{*}, y^{*}\right) \exp \left(i \omega t^{*}\right)
$$

Performing the change of variables $\left(x^{\prime}, y^{\prime}, t^{\prime}\right) \rightarrow\left(x^{\prime *}, y^{\prime *}, \lambda^{*}\right)$ and considering $z^{*} \rightarrow 0$, from (28) and (29) it follows that

$$
\begin{aligned}
V_{0} \frac{\partial h^{*}}{\partial x^{*}}+i \omega h^{*}\left(x^{*}, y^{*}\right)= & \frac{1}{4 \pi V_{0}} \iint_{D^{*}}^{\odot} f\left(x^{*}, y^{*}\right) \exp \left(-\frac{i \omega x_{0}^{*}}{V_{0}}\right) \\
& \times\left[\int _ { - \infty } ^ { x _ { 0 } ^ { * } } \operatorname { e x p } ( \frac { i \omega \lambda ^ { * } } { V _ { 0 } } ) \left(\frac{1}{\left(\lambda^{* 2}+y_{0}^{* 2}\right)^{3 / 2}}\right.\right. \\
& \left.\left.+\frac{8 \chi^{2}-\lambda^{* 2}-y_{0}^{2}}{\left(\lambda^{* 2}+y_{0}^{* 2}+4 \chi^{2}\right)^{5 / 2}}\right) d \lambda^{*}\right] d x^{\prime *} d y^{\prime *}
\end{aligned}
$$

with $x_{0}^{*}=x^{*}-x^{\prime *}, y_{0}^{*}=y^{*}-y^{\prime *}$. The sign $\odot$ indicates the finite part in the Hadamard sense of the integral. Denoting by $a$ the half-span of the wing, we introduce the dimensionless variables

$$
(x, y, z, \lambda, \xi, \eta, \chi, h, t)=\left(\frac{x^{*}}{a}, \frac{y^{*}}{a}, \frac{z^{*}}{a}, \frac{\lambda^{*}}{a}, \frac{x^{\prime *}}{a}, \frac{y^{\prime *}}{a}, \frac{\chi^{*}}{a}, \frac{h^{*}}{a}, \frac{V_{0} t^{*}}{a}\right) .
$$

We reuse the notations $(x, y, z, \lambda, \chi, h, t)$ which must not be confounded with the notations for the dimensional variables corresponding to the fixed frame $O x y z$. Let $D=$ $\left\{(x, y, 0) ;(a x, a y, 0) \in D^{*}\right\}$. The velocity field (with respect to the fixed frame) is $\mathbf{V}=V_{0} \mathbf{i}+\mathbf{v}$, where $\mathbf{v}=u \mathbf{i}+v \mathbf{j}+w \mathbf{k}$ is the perturbation velocity of the fluid. Introducing the dimensionless functions and variables $\tilde{\omega}=\frac{\omega a}{V_{0}}$ (reduced frequency), $\tilde{f}(x, y)=\frac{f(a x, b y)}{V_{0}^{2}}, x_{0}=x-\xi$, $y_{0}=y-\eta$, and taking into account the linearized slip condition, the integral equation (30) becomes

$$
\begin{aligned}
& \frac{\partial h(x, y)}{\partial x}+i \tilde{\omega} h(x, y) \\
& =\frac{1}{4 \pi} \iint_{D}^{\odot} \tilde{f}(\xi, \eta) \exp \left(-i \tilde{\omega} x_{0}\right) \\
& \quad \times\left[\int_{-\infty}^{x_{0}} \exp (i \tilde{\omega} \lambda)\left(\frac{1}{\left(\lambda^{2}+y_{0}^{2}\right)^{3 / 2}}+\frac{8 \chi^{2}-\lambda^{2}-y_{0}^{2}}{\left(\lambda^{2}+y_{0}^{2}+4 \chi^{2}\right)^{5 / 2}}\right) d \lambda\right] d \xi d \eta
\end{aligned}
$$

We discretize the hypersingular integral equation (32) in order to solve it numerically. In Appendix A we split the kernel into several kernels and describe the type of singularity for each one. In Appendix B, depending on the kind of singularity, we deliver appropriate 


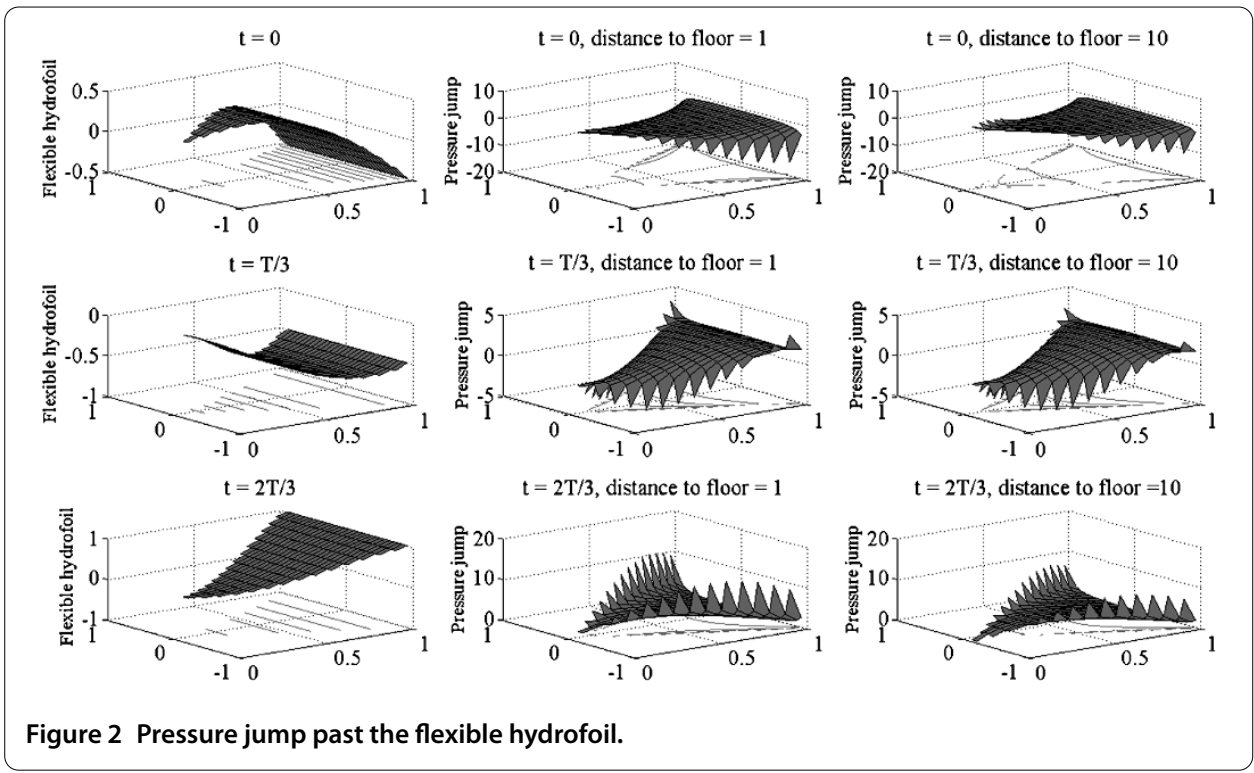

approximation formulas. In order to ensure the uniqueness of the solution, we impose a certain behavior of the pressure jump in the vicinity of the leading edge.

\section{The propulsive force. Numerical results. Floor effects}

We introduce the pressure jump coefficient

$$
C_{p}(x, y, t)=-2 \operatorname{Re}[\tilde{f}(x, y) \exp (i \omega t)] .
$$

We consider the undulatory delta hydrofoil whose equation is

$$
0=z^{*}-\alpha x^{*} \exp \left(i \omega_{1} x^{*}+i \omega t\right) ; \quad\left(x^{*}, y^{*}\right) \in D^{*}
$$

whence, using nondimensional coordinates,

$$
h(x, y)=\alpha x \exp \left(i \tilde{\omega}_{1} x\right), \quad \tilde{\omega}_{1}=a \omega_{1} ; \quad(x, y) \in D .
$$

We used the values $\tilde{\omega}=\pi / 6, \tilde{\omega}_{1}=-2 \pi / 3$ and the nondimensional distances to floor $\chi=1$ and $\chi=10$. In Figure 2 we present (as three-dimensional surfaces with contour plots beneath the surface) the flexible hydrofoil and the pressure jump coefficient fields (divided by $2 \alpha$ ) for the nondimensional moments $t \in\left\{0, \frac{T}{3}, \frac{2 T}{3}\right\}$ where $T=\frac{2 \pi}{\tilde{\omega}}=\frac{2 \pi V_{0}}{a \omega}$ is the nondimensional period of oscillation.

We are also interested in calculating the drag coefficient

$$
C_{D}(t)=\iint_{D} n_{x} C_{p}(x, y, t) d x d y
$$

Since

$$
n_{x}=-\alpha \operatorname{Re}\left[\left(1+i \tilde{\omega}_{1} x\right) \exp \left(i \tilde{\omega}_{1} x+i \tilde{\omega} t\right)\right]
$$




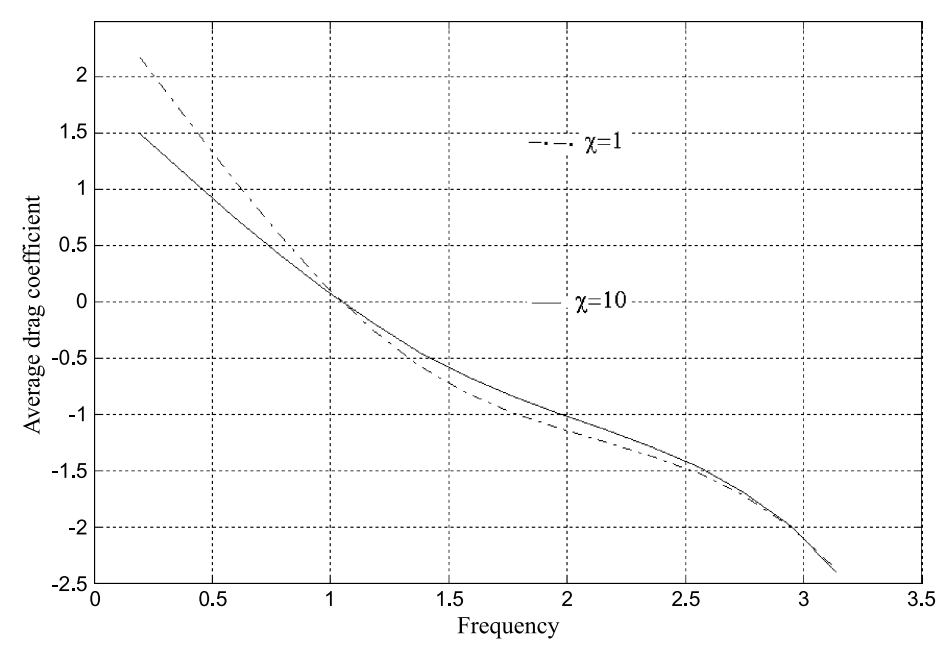

Figure 3 Average drag coefficient for the oscillatory hydrofoil.

we deduce

$$
C_{D}(t)=2 \alpha \iint_{D} \operatorname{Re}\left[\left(1+i \tilde{\omega}_{1} x\right) \exp \left(i \tilde{\omega}_{1} x+i \tilde{\omega} t\right)\right] \operatorname{Re}[\tilde{f}(x, y) \exp (i \tilde{\omega} t)] d x d y
$$

We introduce and we calculate the average drag coefficient:

$$
\begin{aligned}
\tilde{C}_{D} & =\frac{1}{T} \int_{0}^{T} C_{D}(t) d t \\
& =\alpha \iint_{D}\left\{\operatorname{Re}\left[\tilde{f}(x, y) \exp \left(-i \tilde{\omega}_{1} x\right)\right]+\tilde{\omega}_{1} x \operatorname{Im}\left[\tilde{f}(x, y) \exp \left(-i \tilde{\omega}_{1} x\right)\right]\right\} d x d y .
\end{aligned}
$$

In Figure 3 we present the average drag coefficient (divided by $\alpha^{2}$ ) against the reduced frequency for the delta hydrofoil in ground effects. We consider $\tilde{\omega}=\pi / 6, \tilde{\omega}_{1}=-\pi / 2$. We notice that if the reduced frequency surpasses a certain value, the average drag coefficient is negative, i.e. it appears a propulsive force. We also notice that the propulsive force is bigger for $\chi=1$ (dash dot line) than for $\chi=10$ (continuous line) i.e. it is bigger when the oscillatory wing is closer to the floor.

\section{Appendix A: The singularities of the kernel of the integral equation}

For solving numerically the integral equation (32) we have to discretize the left hand member in order to obtain a linear algebraic system of equations. To this aim we split, like in [5] and [6], the kernel

$$
N(x, y ; \xi, \eta)=\int_{-\infty}^{x_{0}} \exp (i \tilde{\omega} \lambda)\left(\frac{1}{\left(\lambda^{2}+y_{0}^{2}\right)^{3 / 2}}+\frac{8 \chi^{2}-\lambda^{2}-y_{0}^{2}}{\left(\lambda^{2}+y_{0}^{2}+4 \chi^{2}\right)^{5 / 2}}\right) d \lambda
$$

into several kernels in order to show the kind of singularities we are dealing with and to find afterwards the most convenient approximation formulas. 
We have step by step

$$
\begin{aligned}
& \int_{-\infty}^{x_{0}} \frac{\exp (i \tilde{\omega} \lambda)}{\left(\lambda^{2}+y_{0}^{2}\right)^{3 / 2}} d \lambda=\int_{-\infty}^{x_{0}} \frac{\exp (i \tilde{\omega} \lambda)-1}{\left(\lambda^{2}+\varpi^{2} y_{0}^{2}\right)^{3 / 2}} d \lambda+\frac{1}{y_{0}^{2}}\left(1+\frac{x_{0}}{\sqrt{x_{0}^{2}+y_{0}^{2}}}\right), \\
& \int_{-\infty}^{x_{0}} \frac{\exp (i \tilde{\omega} \lambda)-1}{\left(\lambda^{2}+y_{0}^{2}\right)^{3 / 2}} d \lambda=\int_{0}^{x_{0}} \frac{\exp (i \tilde{\omega} \lambda)-1}{\left(\lambda^{2}+y_{0}^{2}\right)^{3 / 2}} d \lambda+\int_{0}^{\infty} \frac{\exp (-i \tilde{\omega} \lambda)-1}{\left(\lambda^{2}+y_{0}^{2}\right)^{3 / 2}} d \lambda, \\
& \int_{0}^{\infty} \frac{\exp (-i \tilde{\omega} \lambda)-1}{\left(\lambda^{2}+y_{0}^{2}\right)^{3 / 2}} d \lambda=-\frac{1}{y_{0}^{2}}+\int_{0}^{\infty} \frac{\cos \tilde{\omega} \lambda}{\left(\lambda^{2}+y_{0}^{2}\right)^{3 / 2}} d \lambda-i \int_{0}^{\infty} \frac{\sin \tilde{\omega} \lambda}{\left(\lambda^{2}+y_{0}^{2}\right)^{3 / 2}} d \lambda .
\end{aligned}
$$

The integrals from the right hand part of (43) represent the sine and cosine Fourier transforms of $\left(\lambda^{2}+y_{0}^{2}\right)^{-3 / 2}$ and in [20] one shows that

$$
\begin{aligned}
& \int_{0}^{\infty} \frac{\cos \tilde{\omega} \lambda}{\left(\lambda^{2}+y_{0}^{2}\right)^{3 / 2}} d \lambda=\frac{\tilde{\omega}}{\left|y_{0}\right|} K_{1}\left(\tilde{\omega}\left|y_{0}\right|\right) \\
& \int_{0}^{\infty} \frac{\sin \tilde{\omega} \lambda}{\left(\lambda^{2}+y_{0}^{2}\right)^{3 / 2}} d \lambda=\frac{\pi}{2} \frac{\tilde{\omega}}{\left|y_{0}\right|}\left(L_{-1}\left(\tilde{\omega}\left|y_{0}\right|\right)-I_{1}\left(\tilde{\omega}\left|y_{0}\right|\right)\right) .
\end{aligned}
$$

$L_{-1}, L_{-2}$ are Strouve functions and $I_{1}, I_{2}, K_{1}, K_{2}$ are Bessel functions. We also have

$$
\begin{aligned}
& \int_{0}^{x_{0}} \frac{\exp (i \tilde{\omega} \lambda)-1}{\left(\lambda^{2}+y_{0}^{2}\right)^{3 / 2}} d \lambda \\
& =\int_{0}^{x_{0}} \frac{\exp (i \tilde{\omega} \lambda)-1-i \tilde{\omega} \lambda+\tilde{\omega}^{2} \lambda^{2} / 2}{\left(\lambda^{2}+y_{0}^{2}\right)^{3 / 2}} d \lambda+\frac{i \tilde{\omega}}{\left|y_{0}\right|} \\
& -\frac{i \tilde{\omega}}{\left(x_{0}^{2}+y_{0}^{2}\right)^{1 / 2}}+\frac{\tilde{\omega}^{2} x_{0}}{2\left(x_{0}^{2}+y_{0}^{2}\right)^{1 / 2}}-\frac{\tilde{\omega}^{2}}{2} \ln \left(x_{0}+\sqrt{x_{0}^{2}+y_{0}^{2}}\right)+\frac{\tilde{\omega}^{2}}{2} \ln \left|y_{0}\right|, \\
& \int_{-\infty}^{x_{0}} \exp (i \tilde{\omega} \lambda) \frac{8 \chi^{2}-\lambda^{2}-y_{0}^{2}}{\left(\lambda^{2}+y_{0}^{2}+4 \chi^{2}\right)^{5 / 2}} d \lambda \\
& =\int_{0}^{x_{0}} \exp (i \tilde{\omega} \lambda) \frac{8 \chi^{2}-\lambda^{2}-y_{0}^{2}}{\left(\lambda^{2}+y_{0}^{2}+4 \chi^{2}\right)^{5 / 2}} d \lambda \\
& +12 \chi^{2} \int_{0}^{\infty} \frac{\cos (\tilde{\omega} \lambda)}{\left(\lambda^{2}+y_{0}^{2}+4 \chi^{2}\right)^{5 / 2}} d \lambda-12 i \chi^{2} \int_{0}^{\infty} \frac{\sin (\tilde{\omega} \lambda)}{\left(\lambda^{2}+y_{0}^{2}+4 \chi^{2}\right)^{5 / 2}} d \lambda \\
& -\int_{0}^{\infty} \frac{\cos (\tilde{\omega} \lambda)}{\left(\lambda^{2}+y_{0}^{2}+4 \chi^{2}\right)^{3 / 2}} d \lambda+i \int_{0}^{\infty} \frac{\sin (\tilde{\omega} \lambda)}{\left(\lambda^{2}+y_{0}^{2}+4 \chi^{2}\right)^{3 / 2}} d \lambda, \\
& \int_{0}^{\infty} \frac{\cos (\tilde{\omega} \lambda)}{\left(\lambda^{2}+y_{0}^{2}+4 \chi^{2}\right)^{3 / 2}} d \lambda=\frac{\tilde{\omega}}{\sqrt{y_{0}^{2}+4 \chi^{2}}} K_{1}\left(\tilde{\omega} \sqrt{y_{0}^{2}+4 \chi^{2}}\right) \text {, } \\
& \int_{0}^{\infty} \frac{\cos (\tilde{\omega} \lambda)}{\left(\lambda^{2}+y_{0}^{2}+4 \chi^{2}\right)^{5 / 2}} d \lambda=\frac{2 \tilde{\omega}}{3 \sqrt{y_{0}^{2}+4 \chi^{2}}} K_{2}\left(\tilde{\omega} \sqrt{y_{0}^{2}+4 \chi^{2}}\right) \\
& \int_{0}^{\infty} \frac{\sin (\tilde{\omega} \lambda)}{\left(\lambda^{2}+y_{0}^{2}+4 \chi^{2}\right)^{3 / 2}} d \lambda \\
& =\frac{\tilde{\omega} \pi}{2 \sqrt{y_{0}^{2}+4 \chi^{2}}}\left[L_{-1}\left(\tilde{\omega} \sqrt{y_{0}^{2}+4 \chi^{2}}\right)-I_{1}\left(\tilde{\omega} \sqrt{y_{0}^{2}+4 \chi^{2}}\right)\right],
\end{aligned}
$$




$$
\begin{aligned}
& \int_{0}^{\infty} \frac{\sin (\tilde{\omega} \lambda)}{\left(\lambda^{2}+y_{0}^{2}+4 \chi^{2}\right)^{5 / 2}} d \lambda \\
& \quad=\frac{\tilde{\omega}^{2} \pi}{6\left(y_{0}^{2}+4 \chi^{2}\right)}\left[I_{2}\left(\tilde{\omega} \sqrt{y_{0}^{2}+4 \chi^{2}}\right)-L_{-2}\left(\tilde{\omega} \sqrt{y_{0}^{2}+4 \chi^{2}}\right)\right]
\end{aligned}
$$

From the previous calculations we deduce that we can split the kernel $N(x, y ; \xi, \eta)$ as follows:

$$
N(x, y ; \xi, \eta)=N_{1}(x, y ; \xi, \eta)+\cdots+N_{14}(x, y ; \xi, \eta)
$$

where

$$
\begin{aligned}
& N_{1}=\frac{1}{y_{0}^{2}}\left(\frac{x_{0}}{\sqrt{x_{0}^{2}+y_{0}^{2}}}-\frac{x_{0}}{\left|x_{0}\right|}\right), \quad N_{2}=\frac{1}{y_{0}^{2}}\left(1+\frac{x_{0}}{\left|x_{0}\right|}\right), \quad N_{3}=\frac{-i \tilde{\omega}}{\sqrt{x_{0}^{2}+y_{0}^{2}}}, \\
& N_{4}=\frac{\tilde{\omega}^{2}}{2} \ln \left(\left|y_{0}\right|\right)\left(1+\frac{x_{0}}{\left|x_{0}\right|}\right), \quad N_{5}=-\frac{\tilde{\omega}^{2}}{2} \frac{x_{0}}{\left|x_{0}\right|} \ln \left(\left|x_{0}\right|+\sqrt{x_{0}^{2}+y_{0}^{2}}\right) \text {, } \\
& N_{6}=\frac{\tilde{\omega}^{2} x_{0}}{2 \sqrt{x_{0}^{2}+y_{0}^{2}}}, \quad N_{7}=\frac{\tilde{\omega}}{\left|y_{0}\right|} K_{1}\left(\tilde{\omega}\left|y_{0}\right|\right)-\frac{1}{y_{0}^{2}}-\frac{\tilde{\omega}^{2}}{2} \ln \frac{\tilde{\omega}\left|y_{0}\right|}{2}+\frac{\tilde{\omega}^{2}}{2} \ln \frac{1}{2}, \\
& N_{8}(x, y ; \xi, \eta)=\int_{0}^{x_{0}} \frac{\exp (i \tilde{\omega} u)-1-i \tilde{\omega} u+\tilde{\omega}^{2} u^{2} / 2}{\left(u^{2}+y_{0}^{2}\right)^{3 / 2}} d u, \\
& N_{9}(x, y ; \xi, \eta)=\int_{0}^{x_{0}} \exp (i \tilde{\omega} u) \frac{8 \chi^{2}-u^{2}-y_{0}^{2}}{\left(u^{2}+y_{0}^{2}+4 \chi^{2}\right)^{5 / 2}} d u, \\
& N_{10}=\frac{-\tilde{\omega}}{\sqrt{y_{0}^{2}+4 \chi^{2}}} K_{1}\left(\tilde{\omega} \sqrt{y_{0}^{2}+4 \chi^{2}}\right) \text {, } \\
& N_{11}=\frac{8 \tilde{\omega} \chi^{2}}{\sqrt{y_{0}^{2}+4 \chi^{2}}} K_{2}\left(\tilde{\omega} \sqrt{y_{0}^{2}+4 \chi^{2}}\right) \text {, } \\
& N_{12}(x, y ; \xi, \eta)=\frac{\tilde{\omega} \pi i}{2 \sqrt{y_{0}^{2}+4 \chi^{2}}}\left[L_{-1}\left(\tilde{\omega} \sqrt{y_{0}^{2}+4 \chi^{2}}\right)-I_{1}\left(\tilde{\omega} \sqrt{y_{0}^{2}+4 \chi^{2}}\right)\right], \\
& N_{13}(x, y ; \xi, \eta)=\frac{2 \tilde{\omega}^{2} \pi i \chi^{2}}{\left(y_{0}^{2}+4 \chi^{2}\right)}\left[L_{-2}\left(\tilde{\omega} \sqrt{y_{0}^{2}+4 \chi^{2}}\right)-I_{2}\left(\tilde{\omega} \sqrt{y_{0}^{2}+4 \chi^{2}}\right)\right] \text {, } \\
& N_{14}(x, y ; \xi, \eta)=\frac{i \pi \tilde{\omega}^{2}}{2\left|y_{0}\right|}\left(I_{1}\left(\tilde{\omega}\left|y_{0}\right|\right)-L_{-1}\left(\tilde{\omega}\left|y_{0}\right|\right)+\frac{2}{\pi}\right) \text {. }
\end{aligned}
$$

The integral equation becomes

$$
\begin{aligned}
& \frac{1}{4 \pi} \sum_{i=1}^{14} \iint_{D}^{\odot} \tilde{f}(\xi, \eta) \exp (i \tilde{\omega} \xi) N_{i}(x, y ; \xi, \eta) d \xi d \eta \\
& \quad=\left(\frac{\partial h(x, y)}{\partial x}+i \tilde{\omega} h(x, y)\right) \exp (i \tilde{\omega} x) .
\end{aligned}
$$

The kernels $N_{1}$ and $N_{2}$ have strong singularities of order $\frac{1}{y_{0}^{2}}$. The kernel $N_{3}$ has a polar singularity. The kernels $N_{4}$ and $N_{5}$ have integrable logarithmic singularities. Taking into 
account the series expansions of $L_{-1}, L_{-2}, I_{1}, I_{2}, K_{1}, K_{2}$ we may easily prove that the kernels $N_{7}, N_{10}, N_{11}, N_{12}, N_{13}, N_{14}$ have no singularity and they are continuous functions. $N_{6}, N_{8}$, $N_{9}$ are also continuous functions. We notice that $N_{2}=N_{4}=0$ for $x_{0}<0$.

\section{Appendix B: The discretization of the integral equation}

We consider the undulatory delta hydrofoil. The equations of the leading edge are

$$
y_{ \pm}(x)= \pm \frac{x}{b} ; \quad x \in[0, b]
$$

In order to ensure the uniqueness of the solution of the integral equation, some analytical results from [2] suggest to presume that there exists a continuous finite function $g$ such that $\tilde{f}(\xi, \eta)=\frac{g(\xi, \eta)}{\sqrt{\xi^{2}-b^{2} \eta^{2}}}$. We have therefore

$$
\begin{aligned}
& \iint_{D}^{\odot} \tilde{f}(\xi, \eta) \exp (i \tilde{\omega} \xi) N_{1}(x, y ; \xi, \eta) d \xi d \eta \\
& \quad=F P \int_{-1}^{1} \frac{1}{y_{0}^{2}}\left(\int_{b|\eta|}^{b} \frac{g(\xi, \eta)}{\sqrt{\xi^{2}-b^{2} \eta^{2}}} \exp (i \tilde{\omega} \xi)\left(\frac{x_{0}}{\sqrt{x_{0}^{2}+y_{0}^{2}}}-\frac{x_{0}}{\left|x_{0}\right|}\right) d \xi\right) d \eta
\end{aligned}
$$

where $F P$ stands for the finite part of the hypersingular integral as it is introduced by $\mathrm{Ch}$. Fox in [21]. Since the inner integral vanishes for $\eta=1$, we assume that

$$
\begin{aligned}
& \int_{b|\eta|}^{b} \frac{g(\xi, \eta)}{\sqrt{\xi^{2}-b^{2} \eta^{2}}} \exp (i \tilde{\omega} \xi)\left(\frac{x_{0}}{\sqrt{x_{0}^{2}+y_{0}^{2}}}-\frac{x_{0}}{\left|x_{0}\right|}\right) d \xi \\
& \quad=\sqrt{1-\eta^{2}} G(x, y ; \eta)
\end{aligned}
$$

where $G(x, y ; \eta)$ is finite for $\eta= \pm 1$. We consider on $D$ a net consisting of the nodes (grid points, control points) $\left(x_{h}, \bar{y}_{j}\right)=\left(\frac{h}{n} b, \frac{2 j+1}{2 n}\right), h=1, \ldots, n, j=-h,-h+1, \ldots, h-1$. For the hypersingular integral occurring in (65) we may use the quadrature formula for equidistant control points given by Dumitrescu [22],

$$
\begin{aligned}
& F P \int_{-1}^{1} \frac{\sqrt{1-\eta^{2}} G\left(x_{k}, \bar{y}_{l} ; \eta\right)}{\left(\bar{y}_{l}-\eta\right)^{2}} d \eta=\sum_{j=-n}^{n-1} G\left(x_{k}, \bar{y}_{l} ; \bar{y}_{j}\right) A_{l j}, \\
& A_{l j}=-\arccos \left(y_{j}\right) \\
& +\arccos \left(y_{j+1}\right)+\frac{\sqrt{1-y_{j}^{2}}}{y_{j}-\bar{y}_{l}}-\frac{\sqrt{1-y_{j+1}^{2}}}{y_{j+1}-\bar{y}_{l}}-\frac{\bar{y}_{l}}{\sqrt{1-\bar{y}_{l}^{2}}} \ln \left|\frac{C_{l(j+1)}}{C_{l j}}\right|, \\
& C_{l j}=\frac{\sqrt{1-y_{j}} \cdot \sqrt{1+\bar{y}_{l}}-\sqrt{1+y_{j}} \cdot \sqrt{1-\bar{y}_{l}}}{\sqrt{1-y_{j}} \cdot \sqrt{1+\bar{y}_{l}}+\sqrt{1+y_{j}} \cdot \sqrt{1-\bar{y}_{l}}} .
\end{aligned}
$$

We shall give a quadrature formula for calculating $G\left(x_{k}, \bar{y}_{l} ; \bar{y}_{j}\right)$. Denoting

$$
\bar{x}_{h j}= \begin{cases}x_{h}-\frac{b}{2 n}, & -h<j<h-1, \\ x_{h}-\frac{b}{4 n}, & j \in\{-h, h-1\},\end{cases}
$$




$$
\begin{aligned}
& \tilde{x}_{h j}= \begin{cases}x_{h}-\frac{b}{n}, & -h<j<h-1, \\
x_{h}-\frac{b}{2 n}, & j \in\{-h, h-1\},\end{cases} \\
& g_{h j}=g\left(\bar{x}_{h j}, \bar{y}_{j}\right), \quad H(j)= \begin{cases}1, & j \geq 0, \\
0, & j<0,\end{cases}
\end{aligned}
$$

we have

$$
\begin{aligned}
& \sqrt{1-\bar{y}_{j}^{2}} G\left(x_{k}, \bar{y}_{l} ; \bar{y}_{j}\right) \\
& \simeq \int_{b\left|\bar{y}_{j}\right|}^{b} \frac{g\left(\xi, \bar{y}_{j}\right)}{\sqrt{\xi^{2}-b^{2} \bar{y}_{j}^{2}}} \exp (i \tilde{\omega} \xi)\left(\frac{x_{0}}{\sqrt{x_{0}^{2}+y_{0}^{2}}}-\frac{x_{0}}{\left|x_{0}\right|}\right) d \xi \\
& \simeq \sum_{h=|j|+H(j)}^{n}\left[g_{h j} \exp \left(i \tilde{\omega} \bar{x}_{h j}\right)\left(\frac{x_{k}-\bar{x}_{h j}}{\sqrt{\left(x_{k}-\bar{x}_{h j}\right)^{2}+\left(\bar{y}_{l}-\bar{y}_{j}\right)^{2}}}-\frac{x_{k}-\bar{x}_{h j}}{\left|x_{k}-\bar{x}_{h j}\right|}\right)\right. \\
& \left.\quad \times \int_{\tilde{x}_{h j}}^{x_{i}} \frac{d \xi}{\sqrt{\xi^{2}-b^{2} \bar{y}_{j}^{2}}}\right],
\end{aligned}
$$

whence it follows that

$$
\begin{aligned}
& G\left(x_{k}, \bar{y}_{l} ; \bar{y}_{j}\right) \simeq \sum_{h=|j|+H(j)}^{n} g_{h j} B_{h j k l}, \quad B_{h j k l}=\frac{E_{h j} D_{h j k l}}{\sqrt{1-\bar{y}_{j}^{2}}}, \\
& E_{h j}=\exp \left(i \tilde{\omega} \bar{x}_{h j}\right)\left[\ln \left(x_{h}+\sqrt{x_{h}^{2}-b^{2} \bar{y}_{j}^{2}}\right)-\ln \left(\bar{x}_{h j}+\sqrt{\tilde{x}_{h j}^{2}-b^{2} \bar{y}_{j}^{2}}\right)\right], \\
& D_{h j k l}=\left(\frac{x_{k}-\bar{x}_{h j}}{\sqrt{\left(x_{k}-\bar{x}_{h j}\right)^{2}+\left(\bar{y}_{l}-\bar{y}_{j}\right)^{2}}}-\frac{x_{k}-\bar{x}_{h j}}{\left|x_{k}-\bar{x}_{h j}\right|}\right), \quad-h<j<h-1 .
\end{aligned}
$$

Finally we deduce

$$
\begin{aligned}
& \iint_{D}^{*} \tilde{f}(\xi, \eta) \exp (i \tilde{\omega} \xi) N_{1}\left(x_{k}, \bar{y}_{l} ; \xi, \eta\right) d \xi d \eta \\
& \simeq \sum_{h=1}^{n} \sum_{j=-h}^{h-1} g_{h j} N_{h j k l}^{(1)}
\end{aligned}
$$

with

$$
N_{h j k l}^{(1)}=A_{l j} B_{h j k l} .
$$

For $N_{2}(x, y ; \xi, \eta)=\frac{1}{y_{0}^{2}}\left(1+\frac{x_{0}}{\left|x_{0}\right|}\right)$ we get

$$
\begin{aligned}
& \iint_{D}^{\odot} \tilde{f}(\xi, \eta) \exp (i \tilde{\omega} \xi) N_{2}(x, y ; \xi, \eta) d \xi d \eta \\
& =2 F P \int_{-x}^{x} \frac{1}{y_{0}^{2}}\left(\int_{b|\eta|}^{x} \frac{g(\xi, \eta) \exp (i \tilde{\omega} \xi)}{\sqrt{\xi^{2}-b^{2} \eta^{2}}} d \xi\right) d \eta .
\end{aligned}
$$


Assuming that $\int_{b|\eta|}^{x_{k}} \frac{g(\xi, \eta) \exp (i \tilde{\omega} \xi)}{\sqrt{\xi^{2}-b^{2} \eta^{2}}} d \xi=\sqrt{x_{k}^{2} / b^{2}-\eta^{2}} G^{(k)}\left(x_{k} ; \eta\right)$, we have

$$
\begin{aligned}
& \iint_{D}^{\odot} \tilde{f}(\xi, \eta) \exp (i \tilde{\omega} \xi) N_{2}\left(x_{k}, \bar{y}_{l} ; \xi, \eta\right) d \xi d \eta \\
& =2 F P \int_{-x_{k} / b}^{x_{k} / b} \frac{\sqrt{x_{k}^{2} / b^{2}-\eta^{2}} G^{(k)}\left(x_{k} ; \eta\right)}{\left(\bar{y}_{l}-\eta\right)^{2}} d \eta \simeq 2 \sum_{j=-k}^{k-1} G^{(k)}\left(x_{k} ; \bar{y}_{j}\right) A_{l j}^{(k)}, \\
& A_{l j}^{(k)}=-\arccos \left(\frac{b y_{j}}{x_{k}}\right)+\arccos \left(\frac{b y_{j+1}}{x_{k}}\right) \\
& +\frac{\sqrt{x_{k}^{2}-b^{2} y_{j}^{2}}}{b y_{j}-b \bar{y}_{l}}-\frac{\sqrt{x_{k}^{2}-b^{2} y_{j+1}^{2}}}{b y_{j+1}-b \bar{y}_{l}}-\frac{b \bar{y}_{l}}{\sqrt{x_{k}^{2}-b^{2} \bar{y}_{l}^{2}}} \ln \left|\frac{C_{l(j+1)}^{(k)}}{C_{l j}^{(k)}}\right|, \\
& C_{l j}^{(k)}=\frac{\sqrt{x_{k}-b y_{j}} \cdot \sqrt{x_{k}+b \bar{y}_{l}}-\sqrt{x_{k}+b y_{j}} \cdot \sqrt{x_{k}-b \bar{y}_{l}}}{\sqrt{x_{k}-b y_{j}} \cdot \sqrt{x_{k}+b \bar{y}_{l}}+\sqrt{x_{k}+b y_{j}} \cdot \sqrt{x_{k}-b \bar{y}_{l}}} .
\end{aligned}
$$

For calculating $G^{(k)}\left(x_{k} ; \bar{y}_{j}\right)$ we employ the quadrature formula

$$
\begin{aligned}
G^{(k)}\left(x_{k} ; \bar{y}_{j}\right) & =\frac{1}{\sqrt{x_{k}^{2} / b^{2}-\bar{y}_{j}^{2}}} \int_{b\left|\bar{y}_{j}\right|}^{x_{k}} \frac{g\left(\xi, \bar{y}_{j}\right) \exp (i \tilde{\omega} \xi)}{\sqrt{\xi^{2}-b^{2} \bar{y}_{j}^{2}}} d \xi \\
& =\sum_{i=|j|+H(j)}^{k} g_{i j} \frac{E_{i j}}{\sqrt{x_{k}^{2} / b^{2}-\bar{y}_{l}^{2}}} .
\end{aligned}
$$

At last we find

$$
\begin{aligned}
& \iint_{D}^{*} \tilde{f}(\xi, \eta) \exp (i \tilde{\omega} \xi) N_{2}\left(x_{k}, \bar{y}_{l} ; \xi, \eta\right) d \xi d \eta=\sum_{h=1}^{n} \sum_{j=-h}^{h-1} g_{h j} N_{h j k l}^{(2)}, \\
& N_{h j k l}^{(2)}= \begin{cases}2 A_{l j}^{(k)} \frac{E_{h j}}{\sqrt{x_{k}^{2} / b^{2}-\bar{y}_{l}^{2}}} ; & h \leq k, \\
0 ; & h>k .\end{cases}
\end{aligned}
$$

The singularities of the kernels $N_{3}, N_{4}, N_{5}$ are weaker than $\frac{1}{y_{0}^{2}}$. We replace these kernels with $\frac{N_{3} y_{0}^{2}}{y_{0}^{2}}, \frac{N_{4} y_{0}^{2}}{y_{0}^{2}}, \frac{N_{5} y_{0}^{2}}{y_{0}^{2}}$ for obtaining approximation formulas similar to the formulas for $N_{1}$. We get for $p=3,4,5$ :

$$
\begin{aligned}
& \iint_{D}^{*} \tilde{f}(\xi, \eta) \exp (i \tilde{\omega} \xi) N_{p}\left(x_{k}, \bar{y}_{l} ; \xi, \eta\right) d \xi d \eta \\
& =\sum_{i=1}^{n} \sum_{j=-i}^{i-1} g_{i j} N_{i j k l}^{(p)}, \\
& N_{i j k l}^{(p)}= \begin{cases}\frac{A_{l j}\left(\bar{y}_{l}-\bar{y}_{j}\right)^{2} E_{i j}}{\sqrt{1-\bar{y}_{j}^{2}}} N_{p}\left(x_{k}, \bar{y}_{l} ; \bar{x}_{i j}, \bar{y}_{j}\right) ; & i \neq j, \\
0 ; & i=j .\end{cases}
\end{aligned}
$$


The kernels $N_{6}, N_{7}, N_{8}, N_{9}, N_{10}, N_{11}, N_{12}, N_{13}$, and $N_{14}$ are continuous and we utilize the approximation formulas

$$
\begin{gathered}
\iint_{D}^{*} \tilde{f}(\xi, \eta) \exp (i \tilde{\omega} \xi) N_{p}\left(x_{k}, \bar{y}_{l} ; \xi, \eta\right) d \xi d \eta \\
=\sum_{h=1}^{n} \sum_{j=-h}^{h-1} g_{h j} N_{h j k l}^{(p)}, \quad p=6, \ldots, 14, \\
N_{h j k l}^{(p)}=E_{h j} N_{p}\left(x_{k}, \bar{y}_{l} ; \bar{x}_{h j}, \bar{y}_{j}\right) / n, \quad p=6, \ldots, 14 .
\end{gathered}
$$

For calculating $N_{7}\left(x_{k}, \bar{y}_{l} ; \bar{x}_{h j}, \bar{y}_{l}\right)$ we use the series expansions of the Bessel and Strouve functions and we take into account that

$$
N_{7}\left(x_{k}, \bar{y}_{l}, \bar{x}_{h j}, \bar{y}_{l}\right)=-\frac{\tilde{\omega}^{2}(\psi(1)+\psi(2))}{4}+\frac{\pi i \tilde{\omega}}{4},
$$

where $\psi(1)=-0.5772, \psi(2)=0.4228 . N_{8}\left(x_{k}, \bar{y}_{l} ; \bar{x}_{h j}, \bar{y}_{j}\right)$ and $N_{9}\left(x_{k}, \bar{y}_{l} ; \bar{x}_{h j}, \bar{y}_{j}\right)$ are integrals which are evaluated numerically with the trapezoidal rule. For calculating the Bessel (MacDonald) functions $K_{1}$ and $K_{2}$ we may utilize the series expansions. We may also utilize the libraries offered by MATLAB. For calculating the kernels $N_{12}\left(x_{k}, \bar{y}_{l}, \bar{x}_{h j}, \bar{y}_{j}\right)$ and $N_{14}\left(x_{k}, \bar{y}_{l} ; \bar{x}_{h j}, \bar{y}_{j}\right)$ we use the integral representations

$$
\begin{aligned}
& L_{-1}(x)-I_{1}(x)=\frac{2 \exp (-x)}{\pi}+\frac{2 x}{\pi} \int_{0}^{\pi / 2} \exp (-x \cos t)\left(\sin t-\sin ^{2} t\right) d t \\
& L_{-2}(x)-I_{2}(x) \\
& \quad=-\frac{2}{\pi x}+\frac{2 x \exp (-x)}{3 \pi}+\frac{2 x^{2}}{3 \pi} \int_{0}^{\pi / 2} \exp (-x \cos t)\left(\sin t-\sin ^{4} t\right) d t .
\end{aligned}
$$

The integrals are evaluated numerically with the trapezoidal rule. The approximation formulas for the kernels $N_{1}, N_{2}, N_{6}, N_{7}, N_{8}, N_{9}, N_{10}, N_{11}, N_{12}, N_{13}$, and $N_{14}$ were also given in [5] and [6]. The new approximation formulas for the kernels $N_{3}, N_{4}, N_{5}$ are given for the first time herein. Denoting $N_{h j k l}=N_{h j k l}^{(1)}+N_{h j k l}^{(2)}+\cdots+N_{h j k l}^{(14)}$, we obtain, discretizing the two-dimensional integral equation (32):

$$
\frac{1}{4 \pi} \sum_{h=1}^{n} \sum_{j=-h}^{h-1} g_{h j} N_{h j k l}=\left(\frac{\partial h\left(x_{k}, \bar{y}_{l}\right)}{\partial x}+i \tilde{\omega} h\left(x_{k}, \bar{y}_{l}\right)\right) \exp \left(i \tilde{\omega} x_{k}\right) .
$$

\section{Competing interests}

The authors declare that they have no competing interests.

\section{Authors' contributions}

All authors read and approved the final manuscript.

\section{Author details}

${ }^{1}$ Constanţa Maritime University, Str. Mircea cel Bătrân 104, Constanţa, Romania. ${ }^{2}$ Department of Mathematics, University of Bucharest, Str. Academiei 14, Bucharest, Romania. Institute of Mathematical Statistics and Applied Mathematics of Romanian Academy, Calea 13 Septembrie 13, Bucharest, Romania. 


\section{References}

1. Eloy, C, Doaré, O, Duchemin, L, Scouveiller, L: A unified introduction to fluid mechanics of flying or swimming at high Reynolds number. Exp. Mech. (2009). doi:10.1007/s11340-009-9289-7

2. Carabineanu, A: Incompressible flow past oscillatory wings of low aspect ratio by the integral equations method. Int. J. Numer. Methods Eng. 45, 1187-1201 (1999)

3. Carabineanu, A: Self-propulsion of oscillating wings in incompressible flow. Int. J. Numer. Methods Fluids, 56, 1-21. (2008)

4. Carabineanu, A: Self-propulsion of aquatic animals by undulatory or oscillatory motion. In: Stelian, I, Marinoschi, G (eds.) Proc. 3rd Workshop on Math. Modeling of Environmental and Life Sciences, Constanta, May 2004. Series on Mathematical Modelling of Environmental and Life Sciences Problems, pp. 131-145. Ed. Acad. Romane, Bucharest (2004)

5. Carabineanu, A: Self-propulsion of an oscillatory wing including ground effects. In: Stelian, I, Marinoschi, G, Popa, C (eds.) Proc. 4th Workshop on Math. Modeling of Environmental and Life Sciences, Constanta, September 2005. Series on Mathematical Modelling of Environmental and Life Sciences Problems pp. 39-54. Ed. Acad. Romane, Bucharest (2004)

6. Carabineanu, A: Self-propulsion of an oscillatory wing including tunnel effects. An. Univ. Bucur., Mat. LV, 5-26 (2006)

7. Dowell, EH, Hall, KC: Modeling of fluid-structure interaction. Annu. Rev. Fluid Mech. 33, 445-490 (2001)

8. Dragoş, L: The theory of oscillating thick wings in subsonic flow. Lifting line theory. Acta Mech. 54, $221-238$ (1985)

9. Homentcovschi, D: Theory of the lifting surface in unsteady motion in an inviscid fluid. Acta Mech. 27, 205-216 (1977)

10. Homentcovschi, D: Determination of the vortex associated with the motion of a body in an ideal fluid. Mech. Res. Commun. 3(3), 191-195 (1976)

11. Lighthill, J: Mathematical Biofluiddynamics. SIAM, Philadelphia (1987)

12. Street, A: Preliminary Finite Element Modeling of a Piezometric Actuated Marin Propulsion Fish. Thesis, Department of Mechanical Engineering, Rochester Institute of Technology, Rocester, New York (2006)

13. Taylor, Gl: Analysis of the swimming of long and narrow animals. Proc. R. Soc. Lond. Ser. A 214(117), 158-183 (1952)

14. Wu, TY: Mathematical biofluiddynamics and mechanophysiology of fish locomotion. Math. Methods Appl. Sci. 24, $1541-1564$ (2001)

15. Wu, TY: On theoretical modeling of aquatic and aerial animal locomotion. In: van der Giessen, E, Wu, TY (eds.) Advances in Applied Mechanics, vol. 38, pp. 291-353. Elsevier, Amsterdam (2002)

16. Watkins, CE, Runyan, HL, Woolston, DS: On the Kernel function of the integral equation relating the lift and downwash distributions of oscillating finite wings in subsonic flow. NACA T.R. 1234 (1955)

17. Dragoş, L, Carabineanu, A: A numerical solution for the equation of the lifting surface in ground effects. Commun Numer. Methods Eng. 18, 177-187 (2002)

18. Dragoş, L, Carabineanu, A: The supersonic flow past a thin profile including ground and tunnel effects. Z. Angew Math. Mech. 82, 649-652 (2002)

19. Dragoş, L, Carabineanu, A, Dumitrache, R: A numerical solution for the equation of the lifting line including ground and tunnel effects. Proc. Rom. Acad., Ser. A: Math. Phys. Tech. Sci. Inf. Sci. 9(3), 1-7 (2008)

20. Ditkine, V, Proudnikov, A: Transformations intégrales et calcul opérationnel. Mir, Moscow (1978)

21. Fox, C: A generalisation of the Cauchy principal value. Can. J. Math. 9, 110-115 (1957)

22. Dumitrescu, DF: Three methods for solving Prandtl's equation. Z. Angew. Math. Mech. 76(6), 1-4 (1996)

10.1186/1687-2770-2014-104

Cite this article as: Răpeanu and Carabineanu: A Green function approach for the investigation of the incompressible

flow past an oscillatory thin hydrofoil including floor effects. Boundary Value Problems 2014, 2014:104

\section{Submit your manuscript to a SpringerOpen ${ }^{\circ}$ journal and benefit from:}

- Convenient online submission

- Rigorous peer review

Immediate publication on acceptance

- Open access: articles freely available online

- High visibility within the field

- Retaining the copyright to your article 